\title{
CARACTERIZACION MORFOMETRICA DE LA GERMINACION DE CHLORAEA CRISPA LINDL. (ORCHIDACEAE) USANDO ANALISIS DE IMAGEN
}

\section{MORPHOMETRIC CHARACTERIZATION OF CHLORAEACRISPA LINDL. (ORCHIDACEAE) SEED GERMINATION TROUGH THE IMAGE ANALYSIS TECHNIQUE}

\author{
Gabriela Verdugo ${ }^{1}$, Jorge Marchant ${ }^{1}$, Mauricio Cisternas ${ }^{1}$, \\ Ximena Calderón ${ }^{2} \&$ Patricia Peñaloza ${ }^{1}$. \\ ${ }^{1}$ Facultad de Agronomía, Pontificia Universidad Católica de Valparaíso, Casilla 4 D Quillota \\ ${ }^{2}$ Instituto de Ciencia y Tecnología, Universidad Arturo Prat, Puerto Montt. \\ gverdugo@ucv.cl
}

\section{RESUMEN}

Mediante la técnica de análisis de imagen se evaluó las etapas de germinación de un lote de semillas de Chloraea crispa Lindl. Se pudo observar las cuatro etapas de germinación propuestas por Mitchell. La etapa cero corresponde a semillas sin germinar, éstas son alargadas, curvadas en uno de sus extremos, y alcanzan un largo promedio de $0,5 \mathrm{~mm}$. En la etapa uno se aprecia un incremento sólo en ancho debido al crecimiento del embrión, en la etapa dos hay cambios tanto en la forma como en el aumento del diámetro. En la etapa tres, única visible a simple vista, se inicia la germinación fisiológica. El porcentaje de germinación determinado por esta técnica varió entre 33 y $40 \%$, resultado coincidente con otros autores.

Palabras Claves: Germinación simbiótica, imágenes digitales, orquídeas chilenas.

\section{ABSTRACT}

Through image analysis technique, germination stages of Chloraea crispa Lindl. were evaluated. The four germination steps proposed by Mitchell could be observed. Zero step corresponding to ungerminated seeds, there are lengthened, curved in one point, and $0.5 \mathrm{~mm}$ in mean. In step one just width increase due to embryo growth was observed. In step two there was changing in form and initial diameter was ten times higher. In step three the only marked eye visible, the physiological germination initiates. Germination percentage determined by this technique varied between 33 and $40 \%$.

Keywords: Symbiotic germination, digitals image, Chilean orchids.

\section{INTRODUCCION}

El análisis de imágenes o visión artificial permite recoger datos de manera automática desde imágenes a través de la cuantificación de algunas de sus variables (Kruse 2000). Una imagen de este tipo describe la deducción automática de la estructura y propiedades de un mundo tridimensional a partir de imágenes bidimensionales, se incluyen entonces las propiedades geométricas y estructurales de las imágenes que se analizan. La aplicación requiere de un equipo compuesto por un computador, una cámara o escáner (McDonald et al. 2001) y un procesador digital de imágenes 
Caracterización de la germinación de semillas de Chloraea crispa: Verdugo, G. ET AL

(Maude 2001). Dicha metodología se puede usar con distintos fines, uno de ellos es la identificación de objetos, pues tras el procesamiento, las imágenes son transformadas a lenguaje alfa numérico (Glasbey \& Horgan 1995). La interpretación permite información cuantitativa de elementos básicos que diferencian y dan significado a cada uno de ellos, de este modo, las imágenes sufren transformaciones matemáticas, lo que no se asocia con la calidad estética de las mismas (Maravall 1993).

Esta metodología ha sido descrita como una herramienta potente para cuantificar diversas variables. En experiencias con vegetales se ha determinado una alta correlación con las mediciones manuales (Sako et al. 2001). Otras aplicaciones en semillas son la inspección y clasificación, especialmente de cereales (Sapirstein et al. 1987). También se ha descrito como método complementario a la microscopía en semillas de pequeño tamaño (Glasbey \& Horgan 1995). Otros resultados exitosos incluyen aplicaciones para evaluar la efectividad en tratamientos sanitarios sobre testas, estimación de calidad, dimensiones (Kruse 2000 y Maude 2001) y evaluación de daños (Casady et al. 1992 y Maude 2001), separación de semillas de diferentes especies (Maude 2001), identificación de especies (Kruse 2000), discriminación de variedades (Dehghan-Shoar etal. 1998), madurez (Casady et al.1992) y caracterización de atributos físicos mediante métodos no destructivos (Illipronti et al. 1997).

Las variables frecuentemente evaluadas en la descripción morfológica de semillas mediante análisis de imagen, se centran en aspectos biométricos como la longitud y el ancho (Illipronti et al. 1997; Dehghan-Shoar et al. 1998; Chtioui et al. 1998, Illipronti et al. 1999), el área (Chtioui et al. 1998 y Dehghan-Shoar et al. 1998), la forma (Sahoo et al., 2000) de acuerdo a figuras geométricas o adecuación a modelos matemáticos (Chtioui et al. 1998, Ohsawa et al. 1998, Illipronti et al. 1999), y peculiaridades morfológicas (Dehghan-Shoar et al. 1998). Hay informes de mediciones específicas en testas como estudio del color (Casady et al. 1992, Sahoo et al. 2000), textura (Sahoo et al. 2000) y aspectos tridimensionales (Chtioui et al.1998).

La identificación y caracterización de semillas puede presentar dificultad en función del tamaño y las diferencias morfológicas. El análisis físicobotánico, a pesar de ser casi exclusivamente visual, contiene algunos elementos que mejoran la interpretación (Peretti 1994, Arriagada 2000), pero la observación tiene un valor restringido como herramienta única de diagnóstico (Arriagada 2000). La forma más usual de proceder en la identificación de semillas de cualquier tipo es siguiendo la taxonomía clásica. Sin embargo, es importante considerar que muchos de los descriptores morfológicos pueden estar afectados por factores ambientales (Sahoo et al. 2000). La identificación visual se basa en rasgos filogenéticos (Petetin \& Molinari 1982, Friend 1983, Gunn \& Ritchie 1988, Villaris 1992). Además, se adicionan características de cuantificación específica como la longitud, el ancho, junto a una descripción exhaustiva y detallada de la anatomía propia de cada especie (Berggren 1981).

La identificación de algunos tipos de semillas que presentan alta variabilidad se realiza mediante metodologías de genética molecular (Musselman 1989, Joel et al.1996, Paran et al. 1997), quimiotaxonomía (Endo \& Ohashi 1997), o bioquímica (Velasco et al. 2000). Otras de escaso tamaño (como las pertenecientes al género Orobanche) pudieron ser identificadas alternativamente por la ornamentación de la cubierta (Joel 1987). Estas dificultades plantean el uso de metodologías de apoyo como el análisis de imágenes.

La variable tamaño de las semillas induce a clasificaciones de poca precisión, mientras que la forma distingue mejor pues se asocia más con el genotipo (Sahoo et al. 2000), logra alta discriminación de especies al realizar ajustes matemáticos (Neuman et al. 1987, Sapirstein et al.1987) y se describe como un factor de interés útil para el sistemático (Bengoechea \& Gómez Campo 1975). De este modo, la forma constituye un importante descriptor en la evaluación de recursos genéticos y para discriminar entre los distintos vegetales (Illipronti et al. 1997, Ohsawa et al. 1998).

En este estudio, dado que las orquídeas se caracterizan por poseer semillas muy pequeñas y numerosas, comúnmente llamadas semillas polvo, de considerable variación (Arditti et al. 1980) se ha usado esta técnica para estudiar la germinación de la especie endémica de Chile, Chlorae a crispa Lindl. Con ello se pretende entregar algunos antecedentes que puedan ser usados en la conservación de esta especie cuyo hábitat está fuertemente amenazado 
por plantaciones forestales, en que el manejo tanto de insecticidas como de herbicidas atenta contra la multiplicación de las mismas. Estas semillas están formadas por un embrión de pocas células, (entre 100 y 200), cubiertas por una testa muy dura (Mitchell 1989). El número de semillas puede variar de 13.000 a 4.000.000 por cápsula. El rango de peso de una semilla de orquídea varía de 0,3 a $14 \mu \mathrm{g}$ y miden de 0,25 a 1,2 mm de largo y 0,009 a 0,27 mm de ancho (Arditti 1967). Estas semillas no poseen endosperma y consisten de un pequeño embrión suspendido dentro de una membrana, comúnmente transparente, aunque en ocasiones pigmentada (Arditti 1967, Swamyet al. 2004). Las formas pueden ser muy variables, existiendo elípticas, filiformes, fusiformes, redondas, globulares o prominentemente aladas (Arditti 1967, Mitchell 1989, Molvray \& Kores 1995). Todas estas características aparentemente maximizan la fecundidad y la efectividad en la dispersión por viento de las semillas de orquídeas. A lo menos en los primeros estados de desarrollo presentan una dependencia de una fuente externa de alimentación desde hongos micorrícicos.

En la germinación de las semillas de orquídeas se han descrito cuatro etapas (Mitchell 1989). Etapa 0, semillas sin germinar; Etapa 1, crecimiento del embrión (protocormo) y ruptura de testa; Etapa 2, desarrollo del protocormo y aparición de rizoides; Etapa 3, crecimiento rápido del protocormo y desarrollo de una yema apical. Posterior a esta etapa comienza la aparición de hojas y el desarrollo de raíces que permiten el establecimiento de las plántulas. En especies como Chloraea crispa se cuenta con escasos estudios que describan aspectos de germinación (Mersey 2003), lo que indica la necesidad de describir el proceso y potencial de germinación, contribuyendo a reducir su condición vulnerable.

El tamaño pequeño de las semillas de orquídeas dificulta la caracterización morfológica y fenológica de los primeros eventos del desarrollo simbiótico en plantas mico-heterotróficas (McKendrick et al. 2002). Las actuales técnicas utilizadas para caracterizar la germinación y las etapas posteriores de ésta son realizadas en microscopio de luz y se requiere de mucho tiempo para medir cada una de las muestras.

Es posible pensar que el empleo de análisis de imagen permite identificar y caracterizar las etapas de germinación de las semillas de Chloraea crispa.
El conocer las etapas de germinación podría ayudar a la reproducción sobre todo de aquellas poblaciones que están fuertemente amenazadas por el avance de las plantaciones forestales, especialmente por la pérdida de hábitat y el uso masivo de pesticidas que disminuyen las poblaciones de insectos polinizadores (Novoa et al. 2004). A la fecha se desconoce el estado de conservación de esta especie y de otras orquídeas chilenas (Novoa et al. 2006).

El objetivo de este estudio fue caracterizar morfométricamente las etapas de la germinación de semillas de Chloraea crispa mediante el análisis de imagen.

\section{MATERIALES Y METODOS}

Se utilizaron semillas de Chloraea crispa de ejemplares (o individuos) polinizados manualmente de plantas en cultivo ubicadas en la Estación Experimental La Palma, Quillota (V Región) de la Facultad de Agronomía de la Universidad Católica de Valparaíso.

Se sembraron las semillas inoculadas con la cepa de micorriza denominada MN3 (correspondiente a Rhizoctonia sp. aislada de plántulas de C. crispa) que estimula la germinación en esta especie (Mersey 2003). La siembra se realizó en 25 placas de Petri, (de $9 \mathrm{~cm}$ de diámetro), previa desinfección de las semillas con hipoclorito de sodio al $1 \%$ por 3 minutos (modificado de Clements \& Ellyard 1979). Se preparó un medio en base a agar avena al $0.25 \%$ y se sembró $0,2 \mathrm{mg}$ de semilla por placa, este peso corresponde aproximadamente a un rango entre $110-150$ semillas, las cuales fueron incubadas a $24^{\circ} \mathrm{C}$.

Posteriormente, en la medida que se identificaron a través de lupa las etapas de la germinación propuestas por Mitchell (1989), se extrajo muestras de semillas (Tabla I) que fueron retiradas de 5 placas por etapa para obtener imágenes con 4.800 píxeles de resolución, por medio del escáner Hewlett Packard 7450 tipo "flat bed". Las imágenes obtenidas se procesaron con los programas Photoshop 5.0, y Sigma Scan Pro 5.0, para obtener las características morfométricas de tamaño (área, largo, ancho y volumen). Para evaluar el porcentaje de germinación se consideró semilla germinada a aquella que alcanzó al menos la etapa 1 descrita por Mitchell (1989). 


\section{RESULTADOS Y DISCUSION}

Durante la germinación de Chloraea crispa se observaron diferentes etapas en las cuales se visualizan cambios en la apariencia, el desarrollo y el tiempo de duración, desde semillas con ruptura de testa hasta la formación de protocormos con inicios de una yema apical. Estas etapas son características en especies mico-heterotróficas, especialmente en orquídeas terrestres (Mitchell 1989, McKendrick et al. 2000, McKendrick et al. 2002).

En la etapa 0 las semillas presentan una forma alargada y curvada en uno de sus extremos (Fig. 1A). Esta morfología de las semillas de Chloraea crispa corresponde al tipo "Goodyeroid", la cual es típica para el género Chloraea caracterizado por semillas filiforme y largamente elongadas (Molvray \& Kores 1995). El largo promedio de las semillas fue $0,5 \mathrm{~mm}$, estando dentro del rango $(0,3-0,8 \mathrm{~mm})$ de la mayoría de los taxa estudiados de orquídeas (Arditti et al. 1979, Molvray \& Kores 1995), coincidiendo parcialmente con Arditti (1967), que establece mayores rangos de largo para semillas de orquídeas, lo que puede ser atribuido a la observación exclusivamente visual (Arriagada 2000) o al uso de descriptores inapropiados por su alta variación (Dehghan-Shoar et al. 1998).

La germinación de Chloraea crispa ocurre en los primeros 4-5 días, sin embargo Mersey (2003) señalan que la germinación de Chloraea crispa ocurre 8 días después de la siembra. Esta diferencia pudo ser causada por distintos períodos o condiciones de almacenaje o producto del tiempo de desinfección de las semillas. Mersey (2003) informa cierto deterioro en las semillas dependiendo de dosis y tiempos de desinfección. Esta etapa inicial de la germinación (etapa 1) duró 13 días, momento en que inicia la fase 2. En esta fase se aprecia la ruptura de la testa de las semillas, dejando al descubierto el embrión de color blanquecino, el cual experimenta un crecimiento en ancho al compararlo con la etapa anterior (Fig. 1B, Tabla I). Dicha variación resulta imperceptible al análisis exclusivamente visual.

La etapa 2 se observó a contar de 12 a 13 días después de la siembra y se extendió 14 días aproximadamente, momento en que se observa la etapa 3. En la etapa 2, el protocormo tiene una forma semejante a una elipse, cuyo mayor ancho se ubica en la parte superior. También al final de esta etapa se aprecia el crecimiento de la yema apical, similar a lo descrito por Mitchell (1989), McKendrick et al. (2000) y McKendrick et al. (2002) (Fig. 1C).

La etapa 3 se inició 27 días después de la siembra y se extendió aproximadamente por 31 días. En esta etapa, los protocormos tienen una forma de campana, con un brote en la parte superior (Fig. 1D).

Las etapas 2 y 3 se caracterizan por un crecimiento acelerado del protocormo, permitiendo que las variables compuestas como área y volumen puedan ser utilizadas para caracterizar ambas etapas (Tabla I), como lo describen (Sahoo et al. 2000). A pesar de la poca diferencia de los volúmenes en los dos primeros estados, esta variable puede ser utilizada para distinguir entre semillas germinadas y no germinadas en todos los estados de desarrollo (McKendrick et al. 2000).

Se obtuvieron porcentajes de germinación entre 33 y $40 \%$, en las cinco repeticiones. Esta cifra es concordante con el $48 \%$ que determinó Mersey (2003), utilizando muestras de $0.2 \mathrm{mg}$ y contando campos de observación bajo lupa, lo que podría indicar la germinación natural de la especie y alguna condición endógena no determinada, ya que la falta de conocimientos de los patrones de dormancia en las semillas de esta orquídea no permiten distinguir las semillas de orquideas no viables y aquellas durmientes (Vujanovic et al. 2000). La presencia de mecanismos de dormancia ha sido descrita en orquídeas terrestres de climas templados, relacionándose directamente con la temperatura del ambiente natural donde se desarrolla cada especie (Mitchel 1989, Rasmussen 1992), pero no ha sido descrita para el género Chloraea.

La germinación de semillas de orquídeas, representan el método más eficiente para la propagación de orquídeas nativas para propósitos de conservación (Stewart\& Kane 2006). Sin embargo, a la fecha no se han desarrollado estudios sobre germinación de semillas de otras especies de Chloraea ni géneros afines.

Futuros estudios en germinación de orquídeas nativas permitirían la implementación de estrategias para la puesta en marcha de planes de manejo para la conservación de las orquídeas chilenas a mediano y largo plazo. Especialmente el establecimiento de bancos de semillas que tienen un gran potencial para la conservación de orquídeas, debido al almacenamiento de semillas a largo plazo que permitirá la mantención de la biodiversidad existente (IUCN/SSC 1996). 


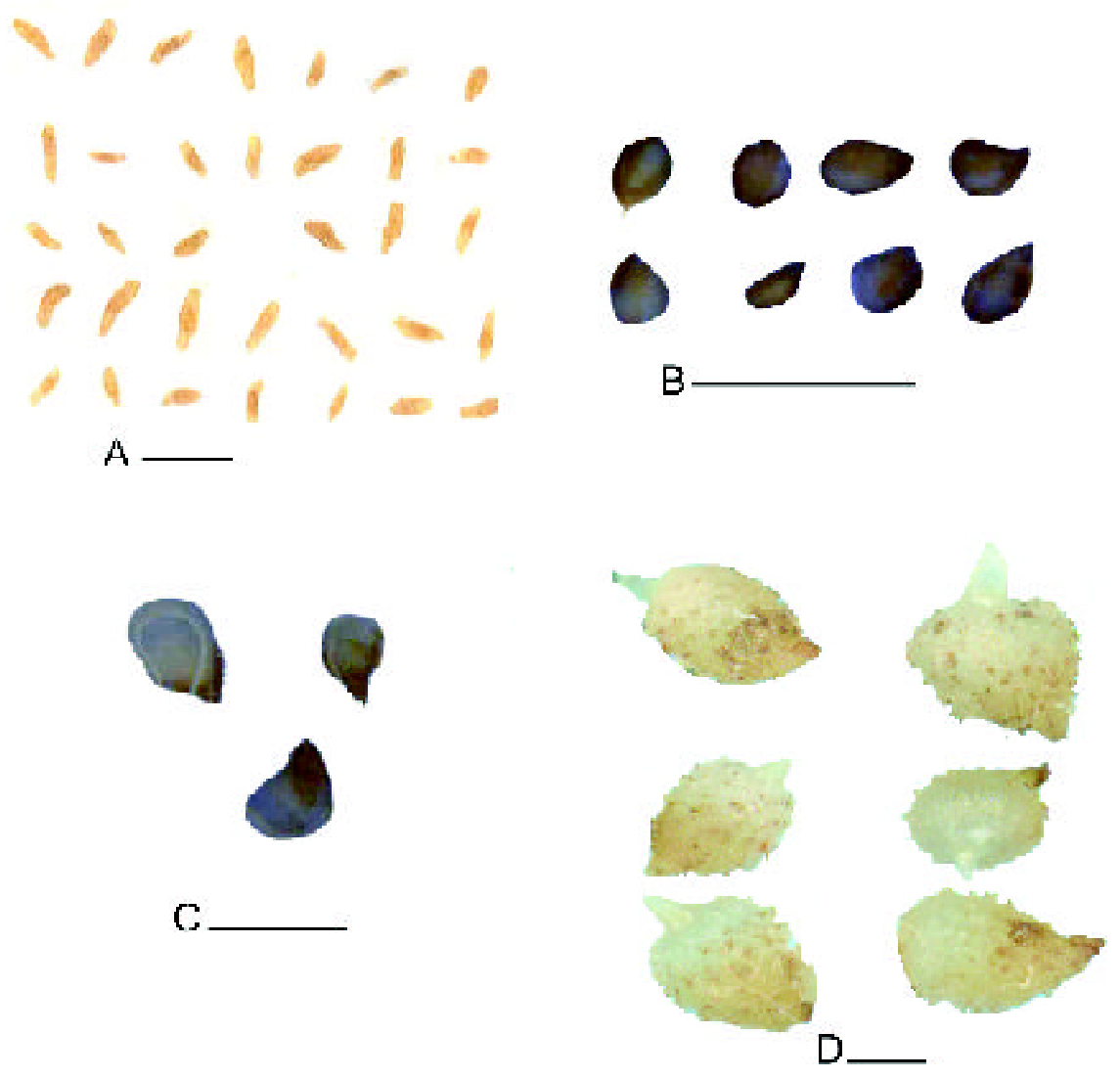

FIgURA 1. Etapas de la germinación de Chloraea crispa: A) Etapa 0: semilla no germinada. B) Etapa 1: semillas hinchadas y rompiendo la testa. C) Etapa 2: inicio de la formación de protocormo y formación de rizoides. D) Etapa 3: protocormo formado con rizoides e iniciando una yema apical. Barras indican $1 \mathrm{~mm}$.

FIGURE 1. Stages during Chloraea crispa seed germination. A) Stage 0: seed without germination. B) Stage 1: seed swollen, starting breaking the testa. C) Stage 2: beginning the protocorms formation and initiating rhizoids. D) Stage 3: protocorm with rhizoids initiating apical buds. Bar $1 \mathrm{~mm}$.

TABLA I. Evaluación de algunos caracteres morfológicos cuantitativos de semillas de Chloraea crispa en germinación.

TABLE I. Evaluation of some quantitative morphological characters on Chloraea crispa germinating seeds.

\begin{tabular}{|c|c|c|c|c|}
\hline \multicolumn{5}{|c|}{ Etapas * } \\
\hline Parámetros & 0 & 1 & 2 & 3 \\
\hline$\overline{\text { Area }\left(\mathrm{mm}^{2}\right)}$ & $0,08 \pm 0,016$ & $0,11 \pm 0,027$ & $0,75 \pm 0,504$ & $2,80 \pm 1,229$ \\
\hline Largo (mm) & $0,53 \pm 0,053$ & $0,52 \pm 0,084$ & $1,23 \pm 0,519$ & $2,49 \pm 0,573$ \\
\hline Ancho (mm) & $0,19 \pm 0,029$ & $0,31 \pm 0,038$ & $0,77 \pm 0,312$ & $1,70 \pm 0,441$ \\
\hline Volumen $\left(\mathrm{mm}^{3}\right)$ & $0,01 \pm 0,003$ & $0,02 \pm 0,006$ & $1,05 \pm 0,841$ & $3,20 \pm 2,382$ \\
\hline $\mathrm{N}$ & 201 & 41 & 81 & 106 \\
\hline
\end{tabular}

* Las etapas según descripción de Mitchell 1989. 
Caracterización de la germinación de semillas de Chloraea crispa: Verdugo, G. et aL.

\section{CONCLUSION}

El método de análisis de imagen permitió estudiar la germinación de semillas de Chloraea crispa, además de describir y cuantificar los cambios morfológicos tanto cualitativos como cuantitativos que sufren durante la germinación. Las variables área, ancho, perímetro y volumen evaluadas permitieron discriminar entre los diferentes estados de la germinación de Chloraea crispa y entre semillas latentes o muertas (sin germinación) respecto a las germinadas.

El análisis de imágenes permite la observación de semillas de escaso tamaño como las de Chloraea crispa, lo que puede colaborar en programas de rescate y reintroducción de materiales a sitios degradados, manteniendo la biodiversidad.

\section{AGRADECIMIENTOS}

A los investigadores del Proyecto FIA C-98-1-A022 .

\section{BIBLIOGRAFIA}

ARDITTI, J. 1967. Factors affecting the germination of orchid seeds. Botanical Review. 33: 1-97.

Arditti, J., D. M ichaud, \& P. Healey. 1979. Morphometry of Orchid Seeds. I. Paphiopedilum and Native California and Related Species of Cypripedium. American Journal of Botany 66: 1128-1137.

Arditti, J., D.Michaud, \& P. Healey. 1980. Morphometry of orchid seeds. II. Native California and related species of Calypso, Cephalanthera, Corallorhiza, and Epipactis. American Journal of Botany 67: 347-360.

Arriagada, R.V. 2000. Semillas Inspección, Análisis, Tratamiento y Legislación. Instituto Interamericano de Cooperación para la Agricultura, Santiago, Chile.114 pp.

Bengoechea, G. \& C. Gómez Campo. 1975. Algunos caracteres de las semillas en la tribu Brassiceae. Anales del Instituto Botánico Antonio José Cavanilles, 32 (2):793-841.

Berggren, G. 1981. Atlas of Seeds and Small Fruits of Northwest-European Plant Species (Sweden, Norway, Denmark, East Fennoscandia and Iceland) with Morphological Descriptions. Part 3, Salicaceae-Cruciferae. Swedish Museum of Natural History, Stockholm. 259 pp.

Casady, W.W., M. R. Paulsen, J.F. Reid \& J.B. Sinclair. 1992. A trainable algorithm for inspection of soybern seed quality. American Society of Agricultural Engineers. 35(6): 2027-2034.

Chtioui, Y., D. Bertrand \& D. Barba. 1998. Feature selection by a genetic algorithm application to seed discrimination by artificial vision. Journal of the Science of Food and Agriculture 76:77-86.

Clements, M. \& R. Elyard. 1979. The symbiotic germination of Australian terrestrial orchids. American Orchid Society Bulletin 48: 810-815.

Dehghan-Shoar, M., J.G. Hampton \& S. J. Haslett. 1998. Identification of, and discrimination among, lucerne (Medicago sativa L.) varieties using seed image analysis. Plant Varieties and Seeds11:107127.

Endo, Y. \& H. Oнаshi.1997 Cladistic analysis of phylogenetic relationships among tribes Cicereae, Trifolieae, and Vicieae (Leguminosae) American Journal of Botany 84:523-529.

Friend, E. 1983. Queensland Weed Seeds. Queensland Departament of Primary Industries, Brisbane. 206 p.

Glasbey, C.A. \& G.W. Horgan. 1995. Image Analysis for the Biological Sciences. John Wiley \& Sons, England. 218 pp.

Gunn, R. C. \& C. A Ritchie. 1988. Identification of Disseminules Listed in the Federal Noxious Weed Act. United States Department Of Agriculture (USDA). $313 \mathrm{pp}$.

ILlipronti JR., R. A., C. J. LANGeraK \& W. J. M. LOMmen. 1997. Variation in and relationships between physical and physiological seed attributes within a soybean seed lot. Seed Science and Technology. 25: $215-231$

Illipronti JR., R.A., C. J. Langerak \& W. J. M Lommen. 1999. Variation in physical seed attributes relates to variation in growth of soybean seedlings within a seed lot. Seed Science and Technology. 27: 339357.

IUCN/SSC Orchid Specialist Group. 1996. OrchidsStatus Survey and Conservation Action Plan. IUCN, Gland Switzerland and Cambridge, UK. $153 \mathrm{pp}$.

JoEL, D. 1987. Detection and identification of Orobanche seeds using fluorescence microscopy. Seed Science and Technology. 15:119-124.

Joel, D., V. Portnoy \& N. K atzir. 1996. Identification of Single Tiny Seed of Orobanche Using RAPD Analysis. Plant Molecular Biology Reporter. 14(3): 243-248.

KRUSE, M. 2000. The effect of moisture content on linear dimensions in cereal seeds measured by image analysis. Seed Science and Technology. 28: 779791

M ARAVALL, D. 1993. Reconocimiento de formas y visión artificial. Madrid. RA-MA Editorial. 433 pp.

M Aude, S.J. 2001. The development of an image analysis technique for the quantitative analysis of seed treatment coverage on seed. Symposium 
Proceedings No 76: Seed treatment: Challenges and opportunities: $55-62$.

McDonald, M. B., A.F. Evans y A.M. Bennett 2001. Using Scanners to Improve Seed and Seedling Evaluations. Seed Sciences and Technology 29: 683-689.

McKendrick, S., J. Leake, D. Taylor \& D. Read. 2000. Symbiotic germination and development of mycoheterotrophic plants in nature: ontogeny of Corallorhiza trifida and characterization of its mycorrhizal fungi. New Phytologist 145:523-537.

McKendrick, S., J. Leake, D. Taylor \& D. Read. 2002. Symbiotic germination and development of the myco-heterotrophic orchid Neottia nidus-avis in nature and its requirement for locally distributed Sebacina spp. New Phytologist. 154(1): 233-247.

Mersey, L. 2003. Diseño y validación de un protocolo para germinación de semillas de Chloraea crispa inoculadas con hongos micorrícicos. Taller de Licenciatura. Ing. Agr. Pontificia Universidad Católica de Valparaíso. Facultad de Agronomía. Quillota. $50 \mathrm{pp}$.

Mitchell, R. 1989. Growing hardy orchids from seeds at Kew. Plantsman 11: 152-169.

Molvray, M \& P. Kores. 1995. Character analysis of the seed coat in Spiranthoideae and Orchidoideae, with special reference to the Diurideae (Orchidaceae). American Journal of Botany 82: 1443-1454.

Musselman, L. 1989. Orobanche ramosa and Orobanche aegyptiaca in Flora Palestina. Progress in Orobanche Research. K. Wegmann und L.J. Musselman Editors, Obermarchtal, FRG. 361 pp.

Neuman, M., H.D Sapirstein, E. Shwedyk \& W. Bushuk. 1987. Discrimination of Wheat Class and Variety by Digital Image Analysis of Whole Grain Samples. Journal of Cereal Science 6: 125-132.

Novoa, P., J. EsPejo \& M.Cisternas. 2004. Iconografía y descripción de las orquídeas nativas de Chile. Chloris chilensis. http://www.chlorischile.cl.

Novoa, P., J. Espejo. M. Cisternas, M. Rubio \& E. Domínguez. 2006. Guía de Campo de las Orquídeas Chilenas. Ed. Corporación Chilena de la Madera. Concepción, Chile. 120 pp.

Ohsawa, R., T. Tsutsumi, H. Uehara, H. Namai \& S. NinomiYa. 1998. Quantitative evaluation of common buckwheat Fagopyrum esculentum
Moench) kernel shape by elliptic Fourier descriptor. Euphytica 101: 175-183.

Paran, I., D. Gidoni \& R. Jacobsohn. 1997. Variation between and within broomrape (Orobanche) species revealed by RAPD markers. Heredity. 78: 68-74.

Peretti, A. 1994. Manual para Análisis de Semillas. Editorial Hemisferio Sur, Buenos Aires. 193 pp.

Petetin, C.\& E. Molinari. 1982. Reconocimiento de Semillas de Malezas. Colección Científica del INTA, Buenos Aires. 146 pp.

Rasmussen, H. N. 1992. Seed dormancy patterns in Epipactis palustris (Orchidaceae), requirements for germination and establishment of mycorrhiza. Physiologia Plantarum 86: 161-167.

Sahoo, L., M. Dadlani, D.P. Singh \& S.P. Sharma. 2000 Characterization of sunflower (Helianthus annuus L.) genotypes using laboratory techniques. Plant Varieties and Seeds. 13: 31-43.

Sako, Y., E. Regnier, T. Daoust, K. Harrison \& M. McDonald. 2001. Computer image analysis and classification of giant ragweed seeds. Weed Science 49: 738-745.

Sapirstein, H.D., M. Neuman, E.H. Wright, E. Shwedyk \&W. Busshuk. 1987. An instrumental system for cereal grain classification using digital image analysis. Journal of Cereal Science 6: 3-14.

Stemart, S. \& M. Kane. 2006. Asymbiotic seed germination and in vitro seedling development of Habenaria macroceratitis (Orchidaceae), a rare Florida terrestrial orchid. Plant Cell Tiss Organ Cult 86: 147-158.

Swamy, K., K. Kumar, T.M. Ramakrishna \& S.N. RaMASWAMY. 2004. Studies on seed morphometry of epiphytic orchids from western ghats of Karnataka. Taiwania 49: 124-140.

Velasco, L., F. Goffman, A. Pujadas-Salva. 2000. Fatty acids and tocochromanols in seeds of Orobanche. Phytochemistry. 54:295-300.

VILLARIS, J.L. 1992. Atlas de "malas hierbas". MundiPrensa, Madrid. 303 pp.

Vujanovic, V., M. St-Arnaud, D. B arabé \& G. Thibeault. 2000. Viability testing of orchid seed and the promotion of colouration and germination. Annals of Botany 86: 79-86. 